\title{
Obstáculos, avatares e implicancias del proceso de incorporación de la población mbya-guaraní en la educación formal de la Provincia de Misiones (Argentina)
}

Yamila-Irupé Nuñez

\section{RESUMEN}

En Argentina, la propuesta de una Educación Intercultural Bilingüe (EIB) surge en 1999 como un enfoque flexible y abierto, dirigido a atender las necesidades de aprendizaje de las poblaciones indígenas, sus intereses y expectativas. No obstante, la EIB se implementó de manera dispar tanto en el tiempo como en la modalidad, con considerables variaciones según la provincia y la población originaria a la que estaba destinada. A partir de las experiencias educativas y laborales de un auxiliar docente indígena (ADI) mbyaguaraní de la Provincia de Misiones (Argentina), este artículo tiene como propósito presentar cómo se llevó a cabo el proceso de incorporación de la población guaraní de Misiones en la educación formal de la sociedad dominante y las implicancias tanto individuales como comunitarias de ello.

Palabras clave: educación intercultural bilingüe, capital intercultural, profesional indígena, auxiliar docente indígena, Argentina. Argentina. Investigadora inicial en la Secretaría de Investigación de la Facultad de Humanidades y Ciencias Sociales de la UNM, Argentina; becaria pos-doctoral del Consejo Nacional de Investigaciones Científicas y Técnicas (CONICET), Argentina. Temas de investigación: antropología y educación: inclusión, interculturalidad y pueblos indígenas; educación superior, pueblos indígenas y nuevos circuitos de mercado laboral; patrimonio cultural / intangible indígena; liderazgo femenino indígena. ORCID: https://orcid.org/0000-0002-0810-7123. 
Obstáculos, avatares e implicações do processo de incorporação da população mbya-guaraní na educação formal da Provincia de Misiones (Argentina)

\title{
RESUMO
}

Na Argentina, a proposta de uma Educação Intercultural Bilingue (EIB) surge em 1999 como um enfoque flexível e aberto, dirigido a atender as necessidades de aprendizagem das populações indígenas, seus interesses e expectativas. Porém, a EIB se implementou de maneira desigual tanto no tempo como na modalidade, com consideráveis variações segundo a província e a população originária à que estava destinada. A partir das experiências educativas e laborais de um auxiliar docente indígena (ADI) mbyaguaraní da Provincia de Misiones (Argentina), este artigo têm como propósito apresentar como realizou o processo de incorporação da população guarani de Misiones na educação formal da sociedade dominante e as implicações tanto individuais como comunitárias disso.

Palavras chave: educação intercultural bilingue, capital intercultural, profissional indígena, auxiliar docente indígena, Argentina.

Obstacles, vicissitudes and implications of the process of incorporating the mbya-guarani population into formal education in the Province of Misiones (Argentina)

\begin{abstract}
In Argentina, the proposal of Intercultural Bilingual Education (IBE) emerged in 1999 as a flexible and open approach, aimed at meeting the learning needs of indigenous populations, their interests and expectations. However, IBE was implemented unevenly both in time and modality, with considerable variations depending on the province and the native population for which it was intended. Based on the educational and work experiences of a mbya-guarani indigenous teaching assistant (ADI) in the Province of Misiones (Argentina), the purpose of this article is to present how the process of incorporation of the Guarani population of Misiones into the formal education of the dominant society was carried out and the individual and community implications of this process.
\end{abstract}

Key words: intercultural bilingual education, intercultural capital, indigenous professional, indigenous teaching assistant, Argentina. 


\section{Introducción}

En relación con la población guaraní, los escritos y documentos del periodo colonial producidos por Cabeza de Vaca, Ulrich Schmidl, Félix de Azara, Montoya, entre otros, entre los siglos XVI al XVIII, favorecieron los estudios etnográficos y etnológicos desarrollados desde comienzos del siglo XX. Entre ellos se destacan los de Branislava Susnik (1965), Nimuendaju (1978), Métraux (1996), Eduardo Viveiros de Castro (1986), Chase Sardi (1972), Müller (1989), Clastres (1993), León Cadogan (1997), Schaden (1974), Melià (1993; 2003; 2012) y Gorosito Kramer (1982; 2004; 2006). Estos estudios trazaron un perfil detallado y comparativo del ethos guaraní y estimularon la posterior generación de investigaciones sobre las poblaciones guaraníes. Todas éstas constituyen referencias ineludibles para el estudio de este grupo étnico. Sin embargo, la dinámica mutable de la realidad social, especialmente en materia de educación y políticas públicas relativas a poblaciones indígenas, demanda la producción constante de nuevas investigaciones empíricas que den cuenta de la profundidad y alcance de estos procesos.

Los guaraníes constituyen uno de los pueblos indígenas de mayor presencia territorial en el continente americano y el territorio ocupado por este grupo étnico es anterior a la creación y conformación de los actuales Estados-nación y sus fronteras. En la actualidad, se reconocen al menos cuatro grandes identidades étnicas ${ }^{1}$ del pueblo guaraní: los mbya-guaraní; los ava katu ete, (ñandéva, o chiripá, como son llamados en Argentina y Paraguay); los paĩ tavyterã, también llamados kaiova; y los aché (aché guajaki en Paraguay). Durante 2015 y 2016 se realizó un relevamiento de comunidades, parajes y barrios guaraníes en Argentina, Bolivia, Paraguay y Brasil a partir del cual se elaboró el Mapa Guaraní Continental (MGC). Dicho relevamiento arrojó un cálculo poblacional de más de 280000 personas para el pueblo guaraní. ${ }^{2}$ Sin embargo, es necesario mencionar que los registros censales de esta población presentan algunas limitaciones debidas a la extensión geográfica de su distribución, así como a las limitaciones presupuestarias para realizar los relevamientos estadísticos, y a la diversidad de situaciones legales en que se encuentran los territorios que ocupan.

Por ello, a menudo se omiten agrupamientos, volviendo las cifras estimativas. En el caso de Argentina, los guaraníes habitan dos regiones bien diferenciadas: la del Litoral, especialmente en la provincia de Misiones, y la de Noroeste, con mayor presencia en las provincias de Salta y Jujuy. En la región del Noroeste se registraron 45000 habitantes distribuidos en 123 comunidades, 69 en la Provincia de Salta y 54 en Jujuy. El grupo más numeroso es el ava-guaraní, aunque se encuentran también los tupi (llamados isoseños en Bolivia), los chané, tapiete y algunas pocas familias zimba (MGG, 2016). Mientras que en la Provincia de Misiones, ${ }^{3}$ la población guaraní alcanza aproximadamente a los 10000 integrantes

\footnotetext{
${ }^{1}$ Esta clasificación original fue desarrollada por el etnólogo brasileño Egon Schaden, no obstante, los criterios utilizados para construir esta taxonomía étnica no están exentos de críticas. Melià (2003) se ha referido a estas etiquetas antropológicas y su vinculación a territorios nacionales como un "muro de Berlín" que mantiene al pueblo guaraní separado y fragmentado.

${ }^{2}$ Que se compone, según el Mapa Guaraní Continental (2016), por las etnias: 1) mbya, presentes en Argentina, Brasil y Paraguay; 2) ava-guaraní (Paraguay), también conocidos como ñandeva, guaraní o chiripá en Brasil y Argentina; 3) paz̃-tavyterã (Paraguay), conocidos como kaiowá en Brasil; 4) ava-guaraní e isoseño en Argentina y Bolivia, respectivamente, también conocidos como guaraní occidental en Paraguay y como chiriguanos o chahuancos en Argentina; 5) gwarayú, sirionó, mbíao yuki, guarasug'we (Bolivia), tapietéo guaraníñandeva (Bolivia, Argentina y Paraguay) yaché (Paraguay).

${ }^{3}$ Se sitúa en el noreste argentino (NE) y se caracteriza por una importante diversidad cultural y lingüística. Gran parte de la población de la provincia interactúa diariamente con la población de los países fronterizos (Paraguay y Brasil), ya sea por cuestiones laborales, comerciales, educativas o porque tienen familiares que viven al otro lado de las fronteras. Los medios de comunicación también contribuyen en esta diversidad, generando un gran impacto en términos culturales y lingüísticos con contenidos que se caracterizan por combinar los idiomas: español, portugués y guaraní, entre otros (Nuñez, 2018a). Asimismo, a partir de 1890 se llevó adelante una política de colonización y de desarrollo agrícola que tenía por objetivo llenar el "vacio" poblacional en el territorio (Schiavoni, 1998; Gallero
} 
distribuidos en 120 tekoa, de los cuales 14 superan los 150 habitantes y sólo uno tiene más de 1000 habitantes (MGG, 2016). Allí, según el criterio de auto-adscripción, los mbya-guaraní constituyen la "etnia guaraní representada mayoritariamente, aun cuando también residen en sus territorios algunos miembros que se reconocen como chiripá (avá katú eté, también designados como ñandéva) y pai tavyterá (también designados como kayová o pañ)" (Gorosito, 2006: 13). Para esta etnia, el territorio es un elemento fundamental para la conformación del tekoa ${ }^{4}$ (su modo de organización sociocultural) y la mantención de su mbya reko (modo de vida).

La base de este modelo de organización es la familia extensa, ${ }^{5}$ que representa la unidad básica de producción, reciprocidad y consumo. Es decir, su modo de organización social se basa en la transmisión de conocimientos a través de las relaciones familiares en sus tekoa. A través de varias generaciones, los guaraníes han sabido transmitir sus prácticas y repertorios culturales con notable eficacia por medio de estrategias propias tales como la oralidad (mediante largas horas de conversación), la participación activa y temprana de los individuos en las actividades del grupo, rituales, entre otras (Arce, 2011). Según Melià, para los guaraníes "el arte de la palabra es el arte de la vida, alma y palabra (ñe'êy) poseen el mismo significado y el portador de un alma estructura su vida para ser soporte y fundamento de las palabras verdaderas" (1995: 31-32). El ayvu rapyta es el fundamento del lenguaje humano para la cultura guaraní y en él descansa el valor otorgado a la palabra hablada. Fue creado por la máxima divinidad $\tilde{N} a m a n d u ~ R u$ Ete

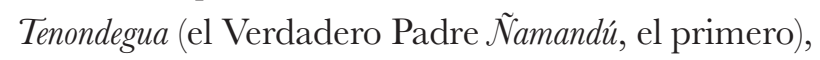

antes de crear la tierra, para que la palabra fuera la verdadera fuente de sabiduría, ligada a la esencia misma del alma (Cadogan, 1997) y después creó el mbae a'ã rapyta petei (himno sagrado). Cuando completó la creación de esos fundamentos, creó los $\tilde{N} e_{e}$ êgy $R u$ Ete (los auténticos padres de las palabras-almas) para transmitir esos principios revelados en el canto sagrado, a los futuros habitantes de la tierra a ser creada: los mbya-guaraní (Martínez Gamba, 1991).

De allí la importancia que se otorga a los y las ancianos/as, considerados/as portadores de las bellas y sabias palabras que se van aprendiendo en el transcurso de la vida y perfeccionando de camino a la vejez (Larricq, 1993). Es en torno de ellos que se organizan las relaciones entre los parientes y son ellos quienes orientan las acciones políticas y religiosas. Es menester destacar que existen discrepancias en relación con la traducción de la palabra ñe'êy que realizaron los autores clásicos en la literatura acerca del pueblo guaraní. En especial, es de vital importancia el trabajo de Ara Rete (Sandra Benites), perteneciente al pueblo guaraní. Según Benites (2015) la concepción de palabra-alma no representa una traducción viable y propone como posible traducción: espíritu-nombre.

La educación guaraní, basada en la oralidad y en rituales, también incorpora la experiencia de todas las etapas de la vida. A través de las palabras que va escuchando y diciendo, y a través de la propia experiencia, la persona guaraní se va consolidando desde su nacimiento, con el acompañamiento de su grupo familiar y su comunidad. Pero si bien la educación guaraní reproduce lo que los adultos reconocen como su "sistema" (su propio modo de vida), esto

2009), con lo cual se recibió población de diferentes lugares de Europa (Bartolomé, 2007). Los impulsores de esta política negaron la presencia de población originaria guaraní, así como de grupos afrodescendientes previamente radicados allí, el resultado fue una estructura social en la que se produjo un marco de interacción caracterizado por relaciones sumamente asimétricas (Nuñez, 2018b).

${ }^{4} \mathrm{El}$ término, también grafiado como tekoha, deriva de tekó entendido como "modo de ser/estar, ley, costumbre, hábito" (Ruiz de Montoya, 1876: 363). El tekoa, que actualmente se traduce literalmente como comunidad o aldea, constituye un concepto complejo que no se reduce al espacio físico de la comunidad sino que corresponde a la estructura básica de la sociedad guaraní, conformada por agrupaciones comunitarias.

${ }^{5}$ Se constituye por grupos macro-familiares que ocupan espacios dentro de los territorios guaraníes, con base en relaciones de afinidad y de consanguinidad. 
ocurre sólo en la medida en que el medio y las condiciones de vida permitan la realización del mismo. En este sentido, todas las formas de la educación guaraní están finamente entretejidas con la práctica social circundante (Larricq, 1993).

\section{Una visión cambiante sobre la educación dominante}

Hasta mediados de la década de los ochenta todavía se rechazaba en la mayoría de las comunidades guaraníes la idea de la escuela y se cuestionaba la necesidad de la escritura como una herramienta de expresión (Gorosito Kramer, 2004). Sin embargo, los cambios producto de la deforestación y el avance de la frontera productiva durante el siglo anterior, y lo que va del actual, han contribuido a alterar la concepción sobre la educación formal en las comunidades guaraníes de la Provincia de Misiones.

$\mathrm{Al}$ reflexionar sobre las transformaciones que la estructura socioeconómica de la sociedad dominante ha producido en su mbya reko, han llegado a ver en la educación de la sociedad dominante un instrumento de poder por medio del cual reivindicar sus derechos y obtener reconocimiento social y cultural. Este quiebre en la actitud de sus líderes con respecto a las escuelas en el seno de las comunidades se dio durante el periodo de 1984 a 1986 (Gorosito Kramer, 2004). Este cambio de actitud tuvo múltiples aristas, entre las que sin duda se destaca la enérgica contribución del jefe guaraní Marçal de Souza, o Marçal Tupã-i, o Tupã-y (pequeño Dios), quien impulsó un fuerte debate al interior de la etnia. La penetrante voz de este líder se hizo escuchar en todas las comunidades a partir de demandar un tipo de escuela con una clara función emancipadora, llegando sus ecos hasta la academia a partir de la grabación realizada por Ana María Gorosito Kramer y su traducción y publicación al español por Carlos Martínez Gamba. A continuación citamos parte de su discurso, publicado en la principal revista paraguaya de antropología en un número homenaje a este líder, que fue brutalmente asesinado el 25 de noviembre de 1983, y condecorado con la honra de héroe nacional de Brasil por el gobierno federal:

"Es necesario que nosotros, es una necesidad, / que hagamos educar a nuestros hijos, / a nuestras hijas, / tenemos que levantar escuelas, / es necesario también que nos provean de remedios, / para mantenernos vigorosos, / para que vivamos sanos. / Esas...Estas cosas, el gobierno tiene que darnos. / [...] Es necesario que nosotros adquiramos fortaleza, / los jóvenes, es necesario/ que adquieran conocimientos, para que se sientan fuertes. / Nosotros, los más viejos, / los que ya no viviremos mañana, / solamente entonces podrán reemplazarnos/ las nuevas juventudes. [...] Es menester que nosotros nos eduquemos; / tal como los señores, / tal como los extranjeros, / tenemos que tener conocimientos para ser fuertes, / para seguir viviendo, / para que nos aseguren nuestras tierras las autoridades poderosas, los extranjeros. [...] Vosotros, los jóvenes, sois más sabios que nosotros, los ancianos. / Vosotros debéis ser la avanzada de nuestras naciones. / Es menester que vosotros trabajéis, / que entabléis continuamente lucha con los grandes jefes, / con los extranjeros, con el gobierno. [...] Eduquemos a nuestros hijos, a nuestras hijas. / Es una necesidad la educación, el ser educados, / para que conozcan las leyes de los extranjeros, / lo que ellos dicen. [...] Y si os otorgan sitios para vuestros emplazamientos/ si os conceden las garantías inherentes a la tierra, / solicitadle al gobierno la erección de escuelas, / para que vuestros hijos y vuestras hijas adquieran sabiduría, / para que sepan vivir por sí mismos/ [...] Os he hablado así brevemente, / qué os quiero decir en nuestro idioma, / Por lo tanto, erguíos y fortaleceos. / Nosotros, a nosotros nos dios Tupä / Nuestro Dios

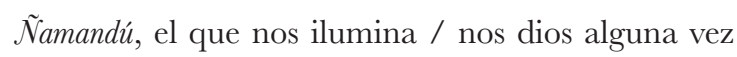
la tierra / Es por eso que nosotros debemos levantarnos, / fortalecernos, para que sigamos viviendo / en nuestros lugares, en nuestras tierras. / en nombre 
de todos los linajes, voy a dejar aquí / mis palabras para que las escuchéis. / En nombre de todos los pueblos indígenas del Brasil, saludos y mucho amor, Marçal de Sousa, Tupã-y. Ese es mi nombre, mi verdadero nombre, Tupã-y" (Martínez Gamba, 1989: 181-200).

Las palabras de Tupã-y definen el núcleo básico de lo que se entiende desde el pueblo guaraní por interculturalidad: "la apertura de los canales de conocimiento que los habiliten para erguirse, para fortalecerse como pueblo en sociedades nacionales que hasta ahora han hecho más por controlarlos que por responder a sus demandas" (Gorosito Kramer, 2004: 75). Desde esta perspectiva, la adopción de la educación de la sociedad dominante constituye una estrategia clave de resistencia étnica.

En este sentido, entendemos que la interculturalidad nace del conflicto de poder que configura procesos y prácticas de diferenciación y subalternización de la sociedad dominante hacia las poblaciones dominadas (pueblos originarios, afrodescendientes, migrantes, por mencionar algunos), y de las estrategias de resistencia que éstas desarrollan (Diez, 2004). Por ello, es necesario pensar las diferencias étnico-culturales en juego como "fenómenos construidos y reproducidos formando parte de una subjetividad y locus de enunciación, definidos por la experiencia de colonización y subalternización social, política y cultural, tanto del pasado como del presente" (Walsh, 2002: 4), y no como diferencias naturales o esenciales de la etnicidad.

En los últimos años, la aceptación de la escuela se ha hecho extensiva a la educación superior, que es la que asegura el acceso a ocupaciones más o menos bien remuneradas, como antaño lo hacia la educación secundaria. Por otra parte, la opción por la educación superior tiene el potencial de convertirse en una oportunidad no sólo para insertarse en un mundo laboral y profesional más amplio, sino también para disponer de autonomía de pensamiento, y manejar herramientas conceptuales y técnicas para contribuir con el desarrollo y la creación del sistema educativo de calidad que las poblaciones indígenas de Misiones demandan. La extensión de la educación entre los varones y mujeres indígenas constituye así la última manifestación del proceso de modernización y expropiación impulsado desde la sociedad dominante, y se presenta como una estrategia clave para resistir, mantener y reivindicar la propia cultura. Sin embargo, los y las estudiantes guaraníes que se insertan en instituciones de educación superior (IES) en la Provincia de Misiones, forjan sus primeras herramientas vinculadas a la educación formal en el seno de familias y comunidades, que se caracterizan por poseer un bajo volumen de capital económico y cultural en relación con los parámetros de las clases dominantes de la sociedad envolvente.

Además de vivir en un medio donde las condiciones materiales son sumamente limitadas, lo que incrementa la dificultad para estudiar en el sistema educativo formal. Por ello, la mayoría de las veces no poseen más alternativa que abandonar los estudios para obtener un trabajo, usualmente informal y mal remunerado. Así, la opción por la educación, en especial de nivel superior, no constituye una estrategia regular y, cuando sí ocurre, es escasamente viable. No obstante su pertenencia a una determinada clase social y grupo étnico, y a las inercias que estas afiliaciones generan en el conjunto de sus estrategias de reproducción, estos/as jóvenes también poseen un margen de construcción de trayectorias propias. Aunque es estadísticamente escaso, la agencia individual o colectiva habilita a veces a abrir espacios que les permiten cambiar las trayectorias típicas por trayectorias propias según su movilidad en el campo social y la acumulación o pérdida de capitales que pongan en juego; lo que Bourdieu denomina como fracciones de clase desviada. Resulta interesante pensar las trayectorias de los y las estudiantes guaraníes que consiguen romper con las tendencias dominantes de sus colectivos en el espacio social como 
parte de este proceso.

Por otro lado, los y las guaraníes que llegan a IES provienen de familias que ocupan posiciones dominantes dentro del ámbito comunitario, y están por lo tanto mejor dotados para acceder y capitalizar esta experiencia; a diferencia de aquellos que nunca tendrán la posibilidad de acceder al sistema educativo superior. Una vez que se insertan en el sistema educativo superior, pasan a conformar un grupo aún más selecto a partir de contar con recursos económicos, culturales y sociales inaccesibles a otros miembros de sus comunidades.

Para pensar esta dinámica resulta particularmente útil el concepto de capital intercultural desarrollado por Andreas Pöllmann (2016a; 2016b; 2017) que lo define como "una forma de capital sociocultural que incluye experiencias, habilidades y competencias interculturales [y] como todo tipo de capital cultural, es de carácter simbólico - incluyendo maneras de ser, ver, actuar y pensar - y provee recursos para lograr el éxito más allá de las formas tradicionales de riqueza económica" (Pöllmann, 2016b: 1). Es decir, esta batería de recursos que Pöllmann denomina capital intercultural, otorga a los y las estudiantes guaraníes mayor reconocimiento que la enorme mayoría de sus pares. Esto a su vez produce nuevas dinámicas y formas de interacción en el interior de sus comunidades y con las principales instituciones estatales con las que se relacionan. El tránsito permanente entre sus comunidades de origen y las IES donde estudian es clave para problematizar las posibilidades y ambiciones de inserción laboral, ya que una vez dentro de ellas, los y las estudiantes guaraníes anticipan la creación de una nueva figura ocupacional: el profesional indígena, que da lugar a la constitución de un nuevo circuito laboral en diversas áreas.

Si bien actualmente se emplean en el área de la educación y la salud, como auxiliares docentes indígenas o agentes sanitarios, el acceso y egreso con éxito de la universidad significa para ellos y ellas la oportunidad inmejorable de disponer de recursos para competir en un mercado laboral mayor al circuito acotado de las comunidades. En este sentido, la emergencia de un nuevo sujeto social indígena (el profesional indígena), producto de la combinación del compromiso étnico-comunitario y la participación en ámbitos universitarios y laborales, puede producir en los próximos años importantes alteraciones en la forma en que se discuten, diseñan y aplican las políticas públicas relativas a la población guaraní y posiblemente a otros sectores de ambas sociedades.

\section{Notas acerca de la metodología utilizada}

En lo que sigue veremos el relato de vida de Crispín con especial referencia a sus experiencias educativas y laborales, para cuya producción trabajamos desde la aplicación de la técnica de entrevista en profundidad. Nos proponemos presentar, a través de su propio relato, cómo se dio el proceso de incorporación de la población guaraní de la provincia Misiones en la educación formal de la sociedad dominante, cuáles fueron sus avatares y las implicancias de dichas transformaciones. Los resultados aquí presentados fueron producidos a partir de una investigación antropológica, acerca del acceso de la población guaraní al sistema educativo superior, de modo que las entrevistas

\footnotetext{
${ }^{6}$ A través de la investigación "Educación superior en contextos de interculturalidad: el caso de los estudiantes guaraníes en la Facultad de Humanidades y Ciencias Sociales (FHCS) de la Universidad Nacional de Misiones (UNM)" que resultaría en mi tesis de licenciatura en antropología social, y de los proyectos de investigación en los que participé: "Pueblos Guaraníes, Gultura y Sociedad" de la Secretaría de Investigación de la FHCS-UNM (2010) y "Territorio, Espacio y Memoria Guaraníes. Dinámicas históricas y procesos culturales en la frontera de Argentina, Brasil y Paraguay" de la Agencia Nacional de Promoción Científica y Tecnológica (2011-2014).

${ }^{7}$ Inicialmente, desde el Proyecto de investigación doctoral CONICET "Mbya-guaraníes y educación. Relaciones interétnicas, interculturalidad y educación universitaria" (2013-2018) y desde 2017 también a través del proyecto de investigación "Inclusión de indígenas, personas portadoras de discapacidad, inmigrantes y de los sectores populares en la universidad", de la Secretaría de Investigación FHCS-UNM, donde coordino el GT Pueblos Indígenas.
} 
fueron desarrolladas en contextos más amplios de investigación etnográfica en dos periodos 2007-2012 ${ }^{6}$ y 2013-2018. ${ }^{7}$ La relación de confianza que fuimos tejiendo a lo largo de esos años nos permitió desarrollar conjuntamente una narración detallada de su vida, haciendo hincapié en su trayectoria educativa y laboral. Para lograrlo fuimos combinando lecturas en conjunto e individuales de las transcripciones de las grabaciones, permitiéndole a nuestro interlocutor ampliar, editar o rectificar sus palabras. El objetivo de este estilo de registro es permitirle a Crispín redactar el guión de su propio relato en un proceso reflexivo, como un intento por desnaturalizar la relación asimétrica entre investigador/investigado (Fals Borda, 1970; 1986; Rappaport, 2007; Kropff, 2005; Díaz y Rodríguez de Anca, 2014) y romper con la monoglosia. A fin de habilitar la distinción entre las voces altas y bajas (Guha, 2002) en el marco de una etnografía dialógica que piense en términos de interculturalidad (Bidaseca, 2010; Gupta, 2014).

\section{La educación de Crispín}

Crispín tiene 39 años, es maestro de educación primaria y auxiliar docente indígena (ADI) en dos escuelas de modalidad intercultural bilingüe ubicadas en el interior de la provincia de Misiones. Fue el segundo egresado guaraní de una institución de educación superior de Misiones y, hasta 2018, el único que residía en la provincia. ${ }^{8}$ Nació en 1979 en el seno de una familia mbya-guaraní proveniente de Garuhapé, que momentáneamente residía en un terreno privado ubicado en Capioví. El propietario del predio lo había cedido para usufructo a un conjunto de familias guaraníes que realizaban diferentes tareas rurales para él. El abuelo materno de Crispín, Bonifacio Ferreyra, provenía de una de las comunidades guaraníes más grandes de la provincia, y era quien organizaba a esas familias. Entre otras tareas, se dedicaban a la tala de árboles, la construcción de caminos, plantación de yerbales y recolección de tung. Diariamente tenían que cubrir 21 kilómetros a pie entre su comunidad y el lugar de trabajo. Éste fue el principal motivo por el que su patrón les concedió una porción de tierra para que pudieran alojarse durante la semana de trabajo. De esta manera, trabajaban durante la semana y los fines de semana regresaban a su comunidad. Debido a que el terreno contaba con una excavación para el abastecimiento de agua potable, las familias se referían a ese lugar como "el pozo".

Con el correr del tiempo el asentamiento fue creciendo a medida que las familias se asentaban allí. El nacimiento de niños y niñas volvió más complicado el traslado semanal hacia su comunidad de residencia. Por este motivo, Bonifacio y su empleador comenzaron a buscar alternativas y, en 1982, consiguieron adquirir por intermedio de la fundación Asociación Indigenista Misionera, un terreno cercano para instalarse definitivamente. Así, Bonifacio dio origen a la comunidad que actualmente se conoce como El Pocito.

Así le decían los de las otras comunidades, Marangatú, Tacuapí y otras, decían "vamos al pozo". Después que se fueron del pozo y al nuevo lugar le llamaron El Pocito. Esa nueva tierra se consiguió por una fundación indigenista que había en esa época, que compraron a través de proyectos. Son 12

\footnotetext{
${ }^{8}$ Previamente hubo otro egresado, el primero de una institución provincial, pero luego de obtener el título de maestro migró hacia Brasil, donde reside actualmente. Asimismo, otro profesional guaraní de Misiones, obtuvo su título de médico en Cuba en 2011 gracias al apoyo de una beca de estudios y se encuentra desarrollando su residencia en Pediatría en el hospital Samic de Oberá, Misiones. En diciembre de 2018 se sumaron otros dos egresados guaraníes, de las carreras Profesorado de Educación Secundaria en Lengua y Literatura, de la extensión áulica del Instituto Superior Antonio Ruiz de Montoya (Eldorado) y de la Tecnicatura en Mantenimiento Edilicio en el Instituto Tecnológico Iguazú (Puerto Iguazú) respectivamente. Por último, este año ha egresado el primer mbya de la carrera de Profesorado en Educación Física del Instituto Carlos Linneo (Oberá, Misiones).
} 
hectáreas nada más la comunidad. En esa época era un terreno grande para la familia de mi abuelo, pero ahora somos más o menos 230 personas.

Tras la fundación de la comunidad, el abuelo de Crispín es elegido como mburuvicha, ${ }^{9}$ posición que mantiene durante más de 30 años. Durante su mandato, asegura Crispín, se consiguieron los principales avances con los que cuenta la comunidad hoy en día, desde el terreno hasta el acceso a agua potable y la construcción de una escuela. "Lo único que no consiguió fue el tendido eléctrico. La escuela es la que nos da la luz, la luz eléctrica llega hasta la escuela".

Unos años después de fundada la nueva comunidad, los padres de Crispín se separan y él se desplaza junto a su padre a la comunidad de Perutí, ubicada en El Alcázar. Por ese entonces, Perutí y Fracrán eran las dos únicas comunidades guaraníes en toda la provincia que contaban con escuelas dentro del predio comunitario, y en ella Crispín tiene la posibilidad de realizar estudios primarios. ${ }^{10}$

A mí siempre me quedó la imagen del director, Ariel, que era mi maestro en séptimo grado. Él me ayudaba. En esa época eran maestros recién recibidos. Se iban con mucho entusiasmo, esa imagen me quedó a mí. Pero nunca pensé que iba estudiar magisterio. Yo soy el único que se recibió de mi familia. Ahora tengo un hermanito de 17 — vive con la otra señora de mi papá- que el año pasado se recibió del secundario. Los otros no terminaron.

Guando cumple 14 años opta por abandonar el núcleo paterno y unirse al grupo doméstico de su madre, que continuaba residiendo en Capioví, con el fin de continuar sus estudios secundarios en la Escuela de la Familia Agrícola (EFA) San Wendelino.

Hice en esa EFA hasta el $3^{\circ}$ año y bueno ahí abandoné totalmente, no quería saber más. Me iba bien hasta tercero, después como que empecé a aflojar. La verdad es que me costaba un poco por las evaluaciones. Porque en la primaria no tenía pruebas, no sé cómo me evaluaban pero miraba mi libreta y estaba todo bien, y cuando entré a la secundaria era otro el ambiente, entonces eso me jugaba en contra. Primer año me costó mucho, y después me fui adaptando. Pero eso más o menos me llevo un año y medio, para que yo me adapte en ese ambiente porque nada que ver con la primaria.

De esa experiencia recuerda que él y otro chico eran los únicos alumnos que provenían de comunidades guaraníes, y que el manejo de la lengua espanola fue una de las principales dificultades que tuvo que enfrentar. A diferencia de la escuela ubicada en Perutí, donde los profesores provenían de Paraguay y, aunque no conocían el mbya, hablaban guaraní paraguayo, ${ }^{11}$ el personal docente de la escuela secundaria era de origen criollo y no tenían manejo alguno de la lengua indígena. Vale mencionar que, aunque la mayor parte de los niños guaraníes comienzan a mantener relaciones laborales con la sociedad envolvente en una edad mucho más temprana que la que Crispín tenía en ese entonces, no es sino hasta que ingresan a la escuela que el contacto con la lengua dominante se torna relevante, especialmente bajo su forma escrita. Fue en esta instancia donde Crispín y su compañero comenzaron

\footnotetext{
${ }^{9}$ Mburuvicha: gran líder. Figura de líder político comunitario mbya-guaraní.

${ }^{10}$ En el "Instituto Hogar Perutí 1207”, escuela rural de gestión privada ubicada dentro de la comunidad, en la que se imparten clases para los niveles inicial, jardín de infantes y primario.

${ }^{11}$ También conocido como guaraní criollo (Cerno, 2013) o avañe’ê (Dietrich 2002: 31; Rodrigues 1984: 38). Según Cerno, surge del proceso de interacción entre españoles e indígenas ocurrido durante la colonización española en las antiguas provincias del Paraguay y Corrientes (siglos XVI-XVIII), y "es una de las ocho lenguas integrantes del grupo de lenguas guaraníes meridionales, según la clasificación de Dietrich (2010:11). Otras lenguas de este grupo son el guaraní jesuítico, el kaiwá (kaiova o Pãa), el ñandeva (chiripá), el mbyá, el xetá, el guaraní chaqueño (chiriguano) y el tapiete" (Cerno, 2013:19).
} 
a adquirir un manejo más fluido del español. Pero a costa de un ejercicio de readaptación lingüística que sus profesores no tenían la inclinación ni la formación para acompañar, y que, por ende, recaía por completo en sus esfuerzos individuales.

Después de la EFA me fui a Loreto. Ahí pase a cuarto año, pero abandoné de nuevo y estuve vagando por ahí unos dos años más. Me iba al yerbal a trabajar, a tarefear, y como que vi que la mano era dura. Ahí me acordaba siempre lo que decía mi viejo: "viste que en la tarefa se sufre mucho". Él me decía que yo tenía la posibilidad de seguir estudiando, de ser un profesional y me acordaba siempre de eso, siempre me quedó en la cabeza. Y por esa razón es que uno empieza a pensar de nuevo y retoma los estudios.

No obstante, durante el cuarto año del secundario, Crispín nuevamente tiene que abandonar sus estudios para dedicarse los siguientes cinco años a complementar el ingreso monetario de su unidad doméstica como tarefero y cosechero. Este recorrido es bastante usual entre los jóvenes guaraníes de la provincia, quienes deben incorporarse desde temprana edad como mano de obra en los puestos peor remunerados del mercado de trabajo rural, trabajando de manera informal en chacras (tareas de limpieza, carpidas, macheteadas, hormigueadas, desyerbado, raleo), montes (desmonte, replantes) y obrajes madereros, entre otros.

Para el año 2008, ${ }^{12}$ el primer registro estadístico específico para población guaraní arrojó que el rubro "empleado rural" agrupaba un 6.6\% de la totalidad de guaraníes de la Provincia de Misiones (cifra que equivale a casi la tercera parte de los artesanos). Este grupo se dividía en 285 individuos (4.8\%) empleados en la tarefa, y $108(1.8 \%)$ que trabajaban como peones en diversas actividades rurales. A este porcentaje, además, hay que sumarles los 134 (2.3\%) guaraníes que indicaban trabajo como changarín en empleos rurales temporarios, principalmente en chacras y plantaciones forestales. De sus años como peón rural, Crispín retiene un consejo paterno.

\begin{abstract}
Mi papá hizo hasta sexto grado y ése era el último año que se hacía antes. Un tiempo trabajó como agente sanitario, pero le quitaron el puesto y tuvo que dedicarse a ser changarín. El consejo que me daba mi papá me quedó en la cabeza: “¿Porqué los guaraníes siempre trabajaron en los yerbales? Hay un sufrimiento importante, hay mucha explotación. Sufrís del frío, pero igual tenés que ir a trabajar". Mi papá me decía: “¿Ves cómo se sufre en el yerbal?”. Nos decía a mí y mi hermano mayor, que también estaba trabajando: "Si ustedes no quieren trabajar sólo tienen que agarrar la birome". Y eso me quedó grabado.
\end{abstract}

$\mathrm{Su}$ oportunidad para escapar de la tarefa llega en el año 2000, cuando tenía 21 años. Durante ese año y el siguiente se realizó un censo complementario para pueblos originarios. Para llevarlo adelante, se contrataron guaraníes con algún grado de capacitación en la educación formal (como ADI o estudiantes). Este requisito abrió la posibilidad para que Crispín se desempeñara como encuestador, una experiencia que produjo en él un renovado interés por la educación formal. En sus palabras, "Me di cuenta de que era importante la educación”. El influjo motivacional provino de un capacitador guaraní de la comunidad de Perutí que estaba a cargo del adiestramiento de los encuestadores. Su forma de dar clases asombró a Crispín, y funcionó como motor para que continuara estudiando.

\footnotetext{
${ }^{12}$ Según el censo desarrollado en el marco del Proyecto Elaboración del Mapa de Tierras y Territorios de los Pueblos Guaraní en Brasil, Argentina y Paraguay, desde el Centro de Trabalho Indigenista (CTI, Brasil), y cuya coordinadora para Misiones (Argentina) fue Ana María Gorosito Kramer.
} 
Aunque le faltaban sólo los dos últimos años del secundario, en aquélla época no había en el interior de Misiones escuelas nocturnas para adultos con modalidad acelerada. Cuando le comunica a la coordinadora del censo su intención de retomar los estudios, ella realiza averiguaciones y le informa que, en Posadas, en el Instituto BAPAYC, ${ }^{13}$ funcionaba un bachillerato de gestión pública de este tipo. El traslado a la capital provincial parecía entonces un recorrido imposible para él, pero habiendo experimentado de primera mano una alternativa económica al padecimiento de vender su fuerza de trabajo como tarefero, estaba decidido a completar su instrucción formal.

Comienza entonces a recorrer diversas instituciones con el objeto de obtener ayuda económica para asentarse en Posadas hasta que, en 2002, recala en la Dirección Provincial de Asuntos Guaraníes. "En esa época ellos (el personal de la institución) no tenían pensado que un guaraní iba querer venir acá a Posadas a seguir estudiando, y les cayó como una sorpresa $^{14}$ porque no se esperaban eso". Una vez superada la sorpresa inicial, la Dirección consiguió gestionar para Crispín un albergue, a través de bienestar social, junto a boletos de transporte público y mercaderías comestibles otorgadas mensualmente.

La iniciativa de Crispín produjo un efecto de bola de nieve y pronto otros jóvenes guaraníes quisieron mudarse a Posadas para terminar sus estudios. Al año siguiente, cuatro estudiantes provenientes de distintas comunidades de la provincia se unieron a él en el albergue. Sin embargo, la vida en la ciudad no era fácil para ellos y los años alejados de la instrucción formal habían implicado la pérdida de hábitos relativos al estudio. A diferencia de los regresos semanales a sus comunidades que caracterizan la dinámica de los estudiantes guaraníes que actualmente cursan estudios de nivel superior, por aquel entonces estos jóvenes debían pasar el mes completo en Posadas, para regresar sólo por unos pocos días a reencontrarse con sus padres, parejas e hijos. Este obstáculo era redoblado por el carácter de proveedor económico que algunos de ellos tenían en sus unidades domésticas, lo que implicaba una búsqueda constante de ingresos monetarios alternativos al que obtenían de sus empleos, si los tuvieran.

El albergue quedaba lejos de la escuela, en $\tilde{N} u$ Porá [Garupá], y no era únicamente para estudiantes guaraníes. En esa época yo ya tenía familia, así que no era fácil. Solamente estudiaba, ya había dejado la tarefa, así que a través del Gobierno, con el PEC [Programa de Empleo Comunitario], pude mantener a mi familia.

La decisión de Crispín y otros guaraníes de retomar los estudios coincide casualmente con la puesta en marcha a nivel nacional de un modelo redistributivo implementado desde el gobierno federal a partir de 2003. Esto los habilitó a ser beneficiarios de una serie de políticas sociales destinadas a personas provenientes de las categorías socioeconómicas más desheredadas del país, que fueron claves para que este grupo de estudiantes pudiera continuar su instrucción formal. En 2007, ${ }^{15}$ el 4.9\% de los guaraníes de la Provincia de Misiones poseía algún tipo de asistencia social, siendo el "Plan Jefes/as de Hogar" el que presentaba mayor concentración, con 111 individuos (1.9\%).

\footnotetext{
${ }^{13}$ Actualmente Escuela Provincial de Educación Técnica No 34.

${ }^{14} \mathrm{La}$ "sorpresa" a la que alude Crispín es indicativa de que en muchas instituciones gubernamentales, y notablemente en aquella que específicamente se encarga de la población guaraní, la prosecución de educación formal en determinados niveles no se prefigura como una alternativa probable, ni siquiera deseable. En 2012, durante una reunión que mantuvimos con la cúpula de esta institución, junto a un grupo de estudiantes guaraníes que solicitaban apoyo económico para continuar sus estudios superiores, un funcionario blanco nos replicó, sorprendido, que nuestra iniciativa iba en contra de los "valores ancestrales de los guaraníes", y que perseguir estudios universitarios implicaría una pérdida de la "forma de vida ancestral" de este grupo étnico, ya que "la universidad es cosa de blancos". ${ }^{15}$ Censo Mapa de Tierras y Territorios de los Pueblos Guaraní en Brasil, Argentina y Paraguay (2008).
} 
En 2004 Crispín consigue egresar del secundario, y ese mismo año opta por inscribirse en la licenciatura de Trabajo Social que ofrece la Facultad de Humanidades y Ciencias Sociales (FHCS) de la Universidad Nacional de Misiones (UNM). Si el paso de la educación primaria a la secundaria había sido difícil para él, el ingreso a la universidad lo fue aún más.

Creo que a los 25 años terminé el secundario. Y quise seguir estudiando, porque yo sé que para ser un profesional hay que pasar por la facultad o universidad y que la secundaria no era la meta [...] Cuando entré en la Facultad de Humanidades yo sentí un cambio más o menos de 180 grados. Me di cuenta que me faltaba formación desde mi base. Me di cuenta de que faltó contenido en la educación primaria y también en la secundaria. Me faltó prepararme [...] Me inscribí en Trabajo Social y bueno, yo creo que por falta de adaptación también dejé, porque duré sólo un mes, y la verdad que me costaba por los horarios. Elegí esa carrera porque pensé que iba ser lo mejor para mi comunidad y para las comunidades de la provincia.

$\mathrm{Al}$ año siguiente vuelve a intentar insertarse en la vida académica pero ésta vez en la licenciatura en Comunicación Social, con el mismo resultado. Durante ese lapso Crispín continuó viviendo en el albergue que la institución provincial había gestionado, complementando sus ingresos con un trabajo en la misma.

Yo seguí viviendo en el albergue porque ayudaba en Asuntos Guaraníes, pero el trabajo no era en blanco. Yo ordenaba y hacía papeles. Trabajaba todos los días en una especie de trabajo administrativo. Y con ese ingreso también me mantenía. Y en ese momento tampoco uno pensaba mucho, si te pagaban no te importaba si era en blanco o en negro el trabajo. Todos trabajamos ahí, todos mis compañeros.
Durante tres años más se mantuvo en esta situación, con un trabajo informal, viviendo en un albergue, e intentando en vano avanzar en una carrera universitaria. Hasta que en 2008 decidió ejercitar una alternativa que le permitiera a la vez obtener un título y conseguir un mejor empleo, sin perder su contacto con su comunidad y su grupo étnico.

Pensé mucho en mi experiencia en la primaria y comencé a pensar también en el futuro de los chicos dentro la comunidad, que en la escuela hace falta un maestro mbya que le enseñe a sus hermanos. Entonces un día me surgió la idea de estudiar magisterio y le comenté al antropólogo que trabajaba en Asuntos Guaraníes, él me mandó hablar con otro antropólogo que trabajaba en EIB. Quien me sugirió que vaya a la Normal Mixta porque tenía una conocida allí, la antropóloga Liliana Seró. Liliana fue mi tutora y la que creó el programa para estudiantes guaraníes ahí.

El programa aludido tenía por objetivo reservar para estudiantes guaraníes algunos de los 150 cupos anuales a los que se accedía por medio de un examen eliminatorio. Durante su primer año de cursado, Crispín notó que tenía dificultad para alcanzar el rendimiento requerido y se sentía desarmado frente a la exigencia y la dinámica de la vida estudiantil urbana. La figura del tutor, que hoy constituye un rasgo fundamental en cualquier programa inclusivo de educación superior para pueblos indígenas, se volvió clave para que Crispín pudiera transitar con éxito su carrera.

En la carrera de formación docente también me costaba un poco. Sobre todo en el primer año, porque todavía no estaba adaptado al nivel superior. En el primer año no tenía amigos ni grupo de estudio, porque no conocía a nadie y me costaba comprender los materiales que me daban para estudiar. Y me di cuenta de que sólo no podía, que necesitaba 
de alguien que me ayude y que esté a mi lado acompañándome. Ahí fue cuando conocí a Liliana S., mi tutora. Gracias a ella empecé a manejarme solo y mi rendimiento mejoró. Me ayudó mucho, me daban una tarea para analizar textos y ya podía hacer solo, ella sólo me corregía.

Y para estudiar hacía resúmenes y cuadros sinópticos y eso ella me enseño y fui incorporando. A mí me gustaban más los exámenes orales porque es como que te podés explayar más. En el escrito es como que no tenés muchas posibilidades. En cambio cuando hablas, oralmente, podés poner ejemplos, y todo eso. A mí también me cuesta un montón escribir pero hablar casi no me cuesta.

Mientras cursaba el primer año de la carrera de magisterio, comenzó a frecuentar habitualmente el área de Educación Intercultural Bilingüe del Instituto de Política Linguística del Ministerio de Cultura, Educación, Ciencia y Tecnología de Misiones. Allí podía solicitar la ayuda de un antropólogo que se desempenaba como técnico de EIB para realizar sus trabajos prácticos. Este contacto regular fue clave para que, en 2008, le ofrecieran incorporarse como pasante en dicha institución. "Y ahí empecé a enterarme de cómo venía la mano, el tema de los ADI. Ya no trabajaba más con Asuntos Guaraníes. Entré como pasante en EIB y hacía tareas de administración, llevaba notas al Consejo y cosas así. Yo acompañaba los cursos que se daban, lo acompañaba a Hugo en las capacitaciones y trabajaba en la oficina de EIB".

Para entonces el programa de Auxiliar Docente Indígena ya se estaba implementando en diversas escuelas del interior. El requisito para trabajar bajo esta modalidad era ser guaraní y tener el secundario completo. Pero a diferencia de los guaraníes empleados en este sistema, Crispín tenía intención de completar su formación superior antes de ingresar al mercado laboral educativo. Sabía que de esta manera tendría mejores posibilidades para hacer carrera, además de producir un cambio sustancial en la dinámica de aprendizaje con niños y niñas guaraníes. Su trabajo como pasante en EIB y los ingresos provenientes de políticas sociales le permitieron solventar parcialmente este anhelo. "Yo pensaba de otra manera, no me quería quedar con el título del secundario nomás. Eso es lo que les pasa a muchos chicos, que terminan e ingresan como ADI y se quedan sin avanzar en sus estudios. Como tienen un ingreso económico ya no quieren estudiar más".

Sin embargo, el camino no estaba allanado todavía. Cuando en 2014 logró finalmente obtener su título, se encontró con que tenía que competir en el mismo mercado que cualquier egresado proveniente de la sociedad dominante, en el que su identidad étnica y su origen de clase perdían su ventaja comparativa para volverse en una clara desventaja (Grimson y Karasik, 2017). De esta manera no le quedó más opción que movilizar su capital intercultural apelando a su identidad como guaraní para conseguir un puesto de ADI en escuelas de modalidad EIB ubicadas en interior de Misiones. Se desempeñó en este puesto durante casi dos años hasta que, a partir de mucha inversión en tiempo, esfuerzo y contactos, logró obtener un trabajo con el perfil para el cual se había formado.

Cuando me recibí no conseguía trabajo porque estaba en el padrón común, no existía una diferenciación ¡C Cuánto tuve que ir a pelear para conseguir eso?! Ahora hay padrones diferentes, hay un padrón para docentes comunes y uno para docentes interculturales, para los docentes que tienen interés en trabajar en escuelas de modalidad EIB”.

Así, en 2015 comenzó a trabajar en una escuela ubicada en Ruíz de Montoya y en aquélla que funciona en la comunidad de El Pocito. $\mathrm{Al}$ inicio dictaba todas las materias y tenía a su cargo primer y segundo año del nivel primario. Esto fue posible debido a que, en razón del bajo número de estudiantes, distintos cursos son agrupados en un mismo salón. Aunque esta decisión depende de la organización interna 
de cada escuela, es un procedimiento habitual en escuelas de modalidad EIB.

Actualmente Crispín reune siete años de antigüedad laboral y es padre de seis hijos, quienes hasta hace poco vivían en Santa Ana Miní. Luego de romper su relación con la madre de sus hijos, se desplazó a la comunidad de Santa Ana junto con sus tres hijos mayores, mientras que los tres más pequeños quedaron con su ex-pareja. Los que viven junto a él asisten a la escuela donde Crispín enseña, y además son sus estudiantes.

Les va bien, y el más grande ya va a primer año. A mí me gustaría que estudien magisterio primero y después de que se reciban pueden seguir estudiando si quieren. No sólo por la salida laboral, sino porque el docente es el que forma a las personas y eso es lo que más falta en las comunidades. Cuando yo me recibí no tuve una respuesta de parte de la comunidad, no es que le dan mucha importancia. Mi familia nomás me festejó. Capaz porque no hay todavía esa conciencia de los mayores de la importancia del título. Y desde mi punto de vista, si se recibe mi hijo ¿Sabes qué? ¡La felicidad!.

Según Crispín, los requisitos para ser ADI fueron cambiando con el correr de los años. Al inicio del proyecto EIB, los postulantes al cargo podían abrir su legajo presentando un certificado que indicara el máximo nivel educativo alcanzado. ${ }^{16}$ La cualificación necesaria para desempeñarse como ADI era la suma de una capacitación ad hoc y los estudios primarios completos. No existía el requisito de secundario completo y como la incorporación de la población guaraní a la educación formal aún era incipiente la gran mayoría de los ADI apenas habían alcanzado el tercero grado de la escuela primaria. También las condiciones laborales experimentaron cambios. "Ahora por resolución del Ministerio, una vez que los ADI presentan los requisitos (estudios psicofísico y demás) pueden titularizarse, se quedan titular en los cargos. Y estamos, desde EIB, preparando para que salga una nueva cohorte de ADI. Ahora son 80 aproximadamente en total, entre los que están y los nuevos".

En relación con los contenidos que brindan los ADI, cada escuela tiene su propia "forma de enseñar" y los contenidos que se imparten son planificados anualmente por el personal docente de cada institución, con cierto grado de articulación ${ }^{17}$ en relación con las autoridades comunitarias. ${ }^{18}$ Esto implica que no hay una homogeneidad curricular entre las escuelas de modalidad EIB de la provincia.

\footnotetext{
16 "Con la Resolución 768 del Ministerio de Cultura, Educación, Ciencia y Tecnología de la Provincia de Misiones (2008) se crea el Área de la Modalidad EIB. y a través del PNEIB se crea la figura del Docente Auxiliar Indígena (ADI), quienes trabajarían en conjunto con los/as docentes no-indígenas como una pareja pedagógica. La capacitación que les brindan consisten en talleres. En el año 2010 con la Ley VI-141 de Planificación Lingüística de la provincia de Misiones se da el marco para la creación del Instituto de Políticas Lingüísticas (IPL), con rango de subsecretaría dentro del Ministerio de Cultura y Educación de Misiones. Actualmente, el IPL presenta dos dispositivos de intervención territorial para la atención educativa intercultural: a) la Modalidad de Educación Intercultural Bilingüe (EIB) destinada al pueblo mbya-guaraní y b) la Modalidad de Educación Intercultural Bilingüe de Frontera (EIBF) que se ocupa, desde el 2005, de las escuelas de localidades fronterizas con Brasil” (Nuñez y Casimiro Córdoba, 2019a: 6-7).

${ }^{17}$ Por ello sería un error generalizar. El grado de articulación depende de cada caso en concreto, de la institución educativa, de los docentes y ADI así como de las figuras de autoridad de cada comunidad. Algunas de las demandas que se plantean son: 1) la falta de materiales pedagógicos y de contenido guaraní para su uso en las aulas; 2) la falta de capacitación de los ADI; 3) la diferencia estructural existente entre maestros no-indígenas y ADI que comparten una misma aula; y 4) la pobre estructura edilicia de la gran mayoría de las escuelas indígenas, entre otras. En relación con el debate de la incorporación de contenidos de la cultura guaraní en el currículo escolar, aquellos/as que se oponen postulan que es necesario que en las escuelas EIB se brinde una "educación de calidad". Ello implicaría que los y las estudiantes puedan incorporar las herramientas necesarias de la educación formal de la sociedad dominante para luego poder competir en los otros niveles educativos, sobre todo en el universitario. En este sentido, exponen que la incorporación de contenido de su cultura en la educación del "blanco" sería redundante para los y las niños/as y jóvenes, pues como pueblo poseen su propio modelo de enseñanza a partir del cual imparten sus saberes tradicionales. Para profundizar al respecto consultar: Nuñez, 2017; Nuñez, 2018a; Nuñez y Casimiro Córdoba, 2019b.

${ }^{18}$ Las autoridades dentro de cada comunidad son: mburuvicha (autoridad política), opyguá (autoridad religiosa) y los/as ancianos/as. Hace unos años se viene dando un proceso de consolidación de liderazgos femeninos en comunidades mbya-guaraní de la Provincia de Misiones, por lo que las mujeres referentes en sus comunidades y las kuña mburuvicha kuery también deben ser consideradas.
} 
En 2011 el Ministerio de Educación desarrolla diseños curriculares jurisdiccionales para el ciclo básico común del secundario obligatorio y del ciclo orientado con carácter diversificado. ${ }^{19} \mathrm{La}$ modalidad EIB debe entonces establecer los lineamientos curriculares ${ }^{20}$ para las escuelas secundarias con matrícula guaraní (Resoluciones 1117/08 y 053/09 MECyT). Sin embargo, en repetidas ocasiones las comunidades guaraníes alertan sobre la desconexión entre la enseñanza escolar y las formas tradicionales de su cultura. En este sentido, los y las ADI que trabajan junto al docente no-guaraní no siempre tienen una materia específica a cargo. "Hay comunidades que están más avanzadas que otras en relación con la enseñanza y los materiales con los que cuentan. Lo que yo quiero hacer es juntar los contenidos de todas las escuelas y que sean parejos, porque cada escuela arma su propio material y hay lindos materiales pero no se distribuyen".

En la práctica, esta falta de homogeneidad y articulación entre escuelas y comunidades en la planificación curricular pone en juego determinadas concepciones relativas a los contenidos de la cultura guaraní, y la valoración sobre cuál es la mejor manera de transmitirla. Mientras que algunas comunidades optan por incorporar contenidos específicos relativos a la forma de vida guaraní, como prácticas relativas a la salud y el cuidado, la historia del pueblo o juegos tradicionales, otras, como en la que enseña Crispín (El Pocito), consideran que la educación tradicional guaraní se realiza fuera de las paredes de una escuela, en el día a día, a través de la oralidad, las actividades cotidianas, los rituales y la experiencia adquirida durante las distintas etapas de la vida de un individuo.

\section{Consideraciones finales}

$\mathrm{Al}$ reconstruir la trayectoria educativa y laboral de Crispín, podemos comprender los cambios producidos en la educación destinada al pueblo guaraní de Misiones. Las primeras escuelas para guaraníes, como mencionamos anteriormente, fueron implementadas por la Iglesia católica con el apoyo del gobierno de turno. Las dos primeras fueron: el Instituto Hogar Perutí 1206 en la Comunidad Perutí (El Alcázar, Departamento San Martín), en 1981 y dos años más tarde el Instituto Paula Mendoza 0805 en la Comunidad Fracrán (San Vicente, Dpto. Guaraní), instituciones que incluso en la actualidad son de subvención estatal pero de gestión privada a través del Obispado. Éstos eran los dos únicos institutos destinados a la población guaraní, de modo que los padres pertenecientes a otras comunidades mbya que deseaban introducir a sus hijos/as en la educación formal debían enviarles a escuelas "comunes", donde la experiencia era mucho más dificil pues sufrían discriminación por parte de docentes y estudiantes.

Impulsados por el Instituto Superior Antonio Ruiz de Montoya (ISPARM), ambos institutos funcionaban bajo la dirección de agentes religiosos y con personal docente de origen paraguayo, cuyo manejo de la lengua indígena era como mínimo diferente, por no mencionar sus concepciones sobre la cultura y la organización social. Asimismo, como brindaban educación únicamente para el nivel educativo primario, aquellos que lograban finalizar sus estudios y deseaban continuarlos debían inscribirse en una escuela "común" lo que implicaba el traslado a zonas urbanizadas. A medida que se fue introduciendo el modelo EIB en la planificación estatal educativa para la población de la Provincia de

\footnotetext{
${ }^{19}$ Resoluciones 638/11 y 048/13 del Ministerio de Cultura, Educación, Ciencia y Tecnología.

${ }^{20}$ Los espacios curriculares son: 1) Lengua y Literatura Mbya-Guaraní, 2) Taller de Integración Curricular Mbya-Guaraní I y II; 3) Taller de Integración Curricular: Producción de textos y Oratorias Mbya-Guaraní I y II; 4) Comunicación Intercultural I y II; 5) Lengua y Narrativa Mbya-Guaraní I y II. Que se desarrollan a través de los ejes transversales: 1) Lengua originaria del Pueblo Mbya-Guaraní; 2) Cultura y Espiritualidad del Pueblo Mbya-Guaraní; 3) Vida y naturaleza en el Pueblo Mbya-Guaraní; 4) Vida social del Pueblo MbyaGuaraní y 5) Leyes y derechos de los Pueblos Originarios.
} 
Misiones, se dio lugar a múltiples enfoques y modelos pedagógicos para atender a la diversidad cultural. Actualmente, en Misiones funcionan 27 escuelas núcleo con la modalidad EIB y 30 aulas satélite (que dependen de escuelas comunes) para el nivel educativo inicial y/o primario. Mientras que para el nivel educativo secundario funcionan apenas seis escuelas núcleo y nueve aulas satélites. Sin embargo, en los discursos de los y las estudiantes mbya-guaraníes universitarios/as y ADI resalta que la calidad educativa brindada en dichas escuelas difiere enormemente de las escuelas de los centros urbanos, resultando de esta manera en otra barrera más a afrontar en su incorporación al sistema educativo formal de la sociedad dominante (Nuñez, 2017). En este sentido, si bien la EIB ha desarrollado avances significativos en relación a la educación brindada a la población indígena y fronteriza de Misiones, aun es necesario que se lleven adelante mejoras sustantivas sobre todo con respecto a su alcance e implicancias en relación con la cultura guaraní.

La historia de Crispín expone algunas de las fragilidades que los estudiantes guaraníes de Misiones experimentan cuando intentan ejercer su derecho a la educación formal y nos permite reflexionar sobre la falta de preparación de las instituciones educativas, en sus diferentes niveles, para recibir y acompañar a estas poblaciones durante su formación. Nos introduce también en la discusión acerca de: a) las relaciones entre los saberes ancestrales guaraníes y la educación formal de la sociedad (o en la ausencia de dichos saberes en la educación formal) y b) la

\section{Referencias}

Arce, H. (2011), "Estrategias y alianzas en el proceso de escolarización guaraní de Misiones, Argentina", en M. Paladino y S. M. García, La escolarización en los pueblos indígenas americanos. Impactos y desafios, Quito, Abya Yala. educación formal y el rol del profesional indígena. En relación con la educación superior, como portador de elementos (históricos, culturales, sociales, cosmológicos, lingüísticos y políticos) distintos de los que comparte la mayoría de la población universitaria, la experiencia de Crispín expone algunas de las carencias de estas instituciones - cristalizadas en preconceptos, prácticas y estrategias pedagógicas estandarizadas - indicando que están mal dotadas para albergar la diferencia. También nos permite apreciar un fenómeno que se viene dando hace pocos años, la emergencia del "profesional indígena". Este nuevo sujeto social se caracteriza por una doble pertenencia, étnica y académica, que tensiona determinados aspectos del sentido común académico. Asimismo, la formación de profesionales indígenas en IES ha contribuido a producir un nuevo circuito laboral, incluso antes de egresar de las mismas, pues al ser considerados "estudiantes universitarios" poseen más chances de conseguir un puesto laboral, por ejemplo como ADI o agente sanitario (las ocupaciones más usuales). Al mismo tiempo, el ingreso de profesionales indígenas en instituciones estatales, con la afirmación de su doble pertenencia (indígena y empleado público), tiene el potencial de alterar las relaciones de poder, las rutinas, las herramientas y los parámetros que las orientan (Pascoal Cassandre, Amaral y Silva, 2016), produciendo en el mediano y largo plazo modificaciones en las perspectivas que orientan los diseños de políticas públicas para estas poblaciones, en especial en relación con la educación formal. 
Fundamento da pessoa guarani, nosso bem-estar futuro (educação tradicional): o olhar distorcido da escola", Monografia final de la Licenciatura Intercultural Indígena do Sul da Mata Atlântica, Florianópolis, Departamento de História, Centro de Filosofia e Ciências Humanas, Universidade Federal de Santa Catarina.

Bidaseca (2010) Perturbando el texto colonial. Los Estudios (Pos) coloniales en América Latina, Bs. As.

Cadogan, L. (1997), Ayvu Rapyta. Textos míticos de los MbyaGuarani del Guairá (3a. ed.) Asunción, Biblioteca Paraguaya de Antropología (Col. Obras de León Cadogan, vol. II, Serie Etnología 1).

Censo Mapa de Tierras y Territorios de los Pueblos Guaraní en Brasil, Argentina y Paraguay (2008) Informe del proyecto de investigación: Pueblos Guaranies. Cultura y Sociedad (16H259), Secretaría de Investigación de la Facultad de Humanidades y Ciencias Sciales de la Universidad Nacional de Misiones.

Cerno, L. (2013), El Guarani Correntino. Fonología, gramática, textos, Frankfurt, Peter Lang GmbH.

Chase Sardi, M. (1972) La situación actual de los indígenas en el Paraguay, Grünberg Coord, pp. 237-307.

Clastres, P. (1993), La palabra luminosa: mitos y cantos sagrados de los guaranies, Buenos Aires, Ediciones Del Sol.

Diaz, R. y A. Rodríguez de Anca (2014), "Activismo intercultural: una mirada descolonizadora, crítica e interseccional", en A. I. Villa y M. E. Martínez (comps.), Relaciones escolares y diferencias culturales: la educación en perspectiva intercultural, Buenos Aires, Editorial Noveduc.

Dietrich, W. (2010), "O tronco tupi e as suas famílias de línguas. Classificação e esboço tipológico", em N. Volker y D. Wolf (eds.), O português e o tupi no Brasil, São Paulo, Contexto.

Dietrich, W. (2002), "Guaraní criollo y guaraní étnico en Paraguay, Argentina y Brasil", en M. Grevels, S. van del Kerde, S. Meira, S y H. van del Voort, (eds.), Current studies on South American languages, Leiden, Research School, GNWS, Universiteit.

Diez, M. L. (2004), "Reflexiones en torno a la interculturalidad", Cuadernos de Antropología Social, núm. 19, pp. 191-213.
Fals Borda, O. (1986), El problema de cómo investigar la realidad para transformarla, Bogotá, Tercer Mundo.

Fals Borda, O. (1970), Ciencia propia y colonialismo intelectual, México, Nuestro Tiempo.

Gallero, M. C. (2009), Con la patria a cuestas. La inmigración alemana-brasileña en la Colonia de Puerto Rico, Misiones, Buenos Aires, Araucaria Editoral.

Gorosito Karmer, A. M. (2006), "Liderazgos guaraníes. Breve revisión histórica y nuevas notas sobre la cuestión”, Avá. Revista de Antropología, núm. 9, pp. 11-27.

Gorosito Karmer, A. M. (2004), "Un debate sobre la interculturalidad", en Educación intercultural bilingüe: debates, experiencias y recursos, Buenos Aires, Ministerio de Educación, Ciencia y Tecnología, Educar.

GorositoKarmer,A.M.(1982), "Encuentrosydesencuentros. Relaciones interétnicas y representaciones en Misiones, Argentina”, Tesis de Maestría, Brasilia, Universidad de Brasília.

Grimson, A. y A. Karasik (2017), Estudios sobre diversidad sociocultural en la Argentina contemporánea, Buenos Aires, CLACSO, PISAC.

Guha, R. (2002), Las voces de la historia y otros estudios subalternos, Barcelona, Crítica.

Gupta, A. (2014), "Authorship, research assistants and the ethnographic field", Ethnography, vol. 15, pp. 394-400.

Kropff, L. (2005), Activismo mapuche en Argentina: trayectoria histórica y nuevas Propuestas, Buenos Aires, CLACSO.

Larricq, M. (1993), Ipytuma. Construcción de la persona entre los Mbya-Guarani, Posadas, Editorial Universitaria UNaM.

Martínez Gamba, C. (1991), El canto resplandeciente. Ayvu Rendy Vera. Plegarias de los mbya-guaraní de Misiones, Buenos Aires, Ediciones del Sol.

Melià, B. (2012), "La interculturalidad y la farsa del bilingüismo", Revista Abehache, núm. 2, pp. 89-94.

Melià, B. (2003), La lengua guarani en el Paraguay colonial, Asunción, CEPAG.

Melià, B. (1993), El guaraní conquistado y reducido. Ensayos de etnohistoria, Asunción, Biblioteca Paraguaya de Antropología (5).

Metraux, A. (1996), Etnografia del Chaco (edición, exordio, revisión y notas a cargo de M. Chase-Sardi), Paraguay, 
Editorial El Lector.

MGG (2016) Mapa Guarani Continental. Cuaderno Mapa Guarani Continental: pueblos Guaranies en Argentina, Bolivia, Brasily Paraguay, Campo Grande, Mundial.

Müller (1989) Etnografia de los guarani del alto parana, Buenos Aires, Centro Argentino de Etnologia Americana.

Nimuendajú, C. U. (1978), Los mitos de creacióny de destrucción del mundo como fundamento de la religión de los ApapokuvaGuarani, Lima, Centro Amazónico de Antropología y Aplicación Práctica.

Nuñez, Y. I. (2018a), "Estudiantes mbya-guaraní y educación intercultural bilingüe en la Provincia de Misiones (Argentina)", Revista Estudio (Dossier temático, Trayectorias juveniles: familia, educación y empleo), núm. 26.

Nuñez, Y. I. (2018 b), "Legislación y programas destinados a la inclusión de guaraníes en la educación superior de la provincia de Misiones (Argentina)", en A. Salete Loss y P. V. Vain, Ensino superior e inclusão: palavras, pesquisas e reflexões entre movimentos internacionais, Curitiva, Brasil, CRV, pp. 397-416.

Nuñez, Y. I. (2017), "Estudiantes guaraníes, interculturalidad y educación superior en Misiones, Argentina", Comparative Cultural Studies-European and Latin American Perspectives (Dossier temático, Interculturalid(es) y racismo(s): aproximaciones críticas y distintas gramáticas de gestión de la diversidad), vol. 2, núm. 4, pp. 9-22.

Nuñez, Y. I. y Casimiro Córdoba, A. V. (2019 a), "Educación intercultural bilingüe. Reflexiones acerca de los casos de Salta y Misiones (Argentina)", Revista Administración Pública y Sociedad (Dossier temático: Política y Derecho Indígena), núm. 8, pp. 178-197.

Nuñez, Y. I. y A. V. Casimiro Córdoba (2019b), "La educación intercultural bilingüe y sus desafíos para población guaraní de Salta y Misiones (Argentina)", Revista Mexicana de Investigación Educativa (RMIE), vol. 25, núm. 85, pp. 419-447.
Pascoal Cassandre, Amaral y Silva (2016) "Eu, Alex, da etnia Guarani: o testemunho de um estudante indígena de administração e seu duplo pertencimento", Cadernos EBAPE.BR, vol. 14, núm. 2, Rio de Janeiro.

Pöllmann, A. (2017), "Miradas a las culturas indígenas mexicanas desde la perspectiva de futuros maestros de secundaria”, Universidady Sociedad, núm. 9, pp. 53-58.

Pöllmann, A. (2016a), "Habitus, reflexividad y la realización de capital intercultural: El potencial (no aprovechado) de la educación intercultural", Cultura y representaciones sociales, núm. 11, pp. 55-78.

Pöllmann, A. (2016b), "Capital intercultural”, Key Concepts in Intercultural Dialogue, núm. 6, pp. 1-2.

Rappaport (2007) "Más allá de la escritura: la epistemología de la etnografía en colaboración", Revista Colombiana de Antropología, vol. 43, enero-diciembre, pp. 197-229, Instituto Colombiano de Antropología e Historia Bogotá, Colombia.

Rodrigues, A. D. (1984), "Relações internas na familia lingüística Tupí-Guaraní”, Revista de Antropología, núm. 28, pp. 33-53.

Ruiz de Montoya (1876) Tesoro de la Lengua Guarani, Leipzig, B.G.Teubner.

Schaden, E. (1974), Aspectos fundamentais da cultura guarani, San Pablo, EDFUSP.

Schiavoni, G. (1998), Colonos y ocupantes. Parentesco, reciprocidad y diferenciación en la frontera agraria de Misiones, Posadas, Editorial Universitaria.

Susnik, B. (1965), El indio colonial del Paraguay. El guaraní colonial, Asunción, Museo Etnográfico Andrés Barbero.

Viveiros de Castro, E. (1986), Araweté os deuses canibais, Rio de Janeiro, ANPOCS.

Walsh, G. (2002), "(De)Construir la interculturalidad. Consideraciones críticas desde la política, la colonialidad y los movimientos indígenas y negros en el Ecuador", en N. Fuller (ed.), Interculturalidad y política, Lima, Red de Apoyo de las Ciencias Sociales.

\section{Cómo citar este artículo:}

Nuñez, Yamila-Irupé (2021), “Obstáculos, avatares e implicancias del proceso de incorporación de la población mbya-guaraní en la educación formal de la Provincia de Misiones (Argentina)", Revista Iberoamericana de Educación Superior (RIES), vol. XII, núm. 35, pp. 206-223, DOI: https://doi.org/10.22201/iisue.20072872e.2021.35.1090 [Consulta: fecha de última consulta]. 